\title{
Upregulation of vitamin D-related genes in schizophrenic patients
}

This article was published in the following Dove Press journal:

Neuropsychiatric Disease and Treatment

\author{
Fateme Asadzadeh Manjili' \\ Seyed Mehdi Kalantar ${ }^{2}$ \\ Shahram Arsang-Jang ${ }^{3}$ \\ Soudeh Ghafouri-Fard ${ }^{4}$ \\ Mohammad Taheri ${ }^{4,5}$ \\ Arezou Sayad ${ }^{4}$
}

'Department of Medical Genetics, Shahid Sadoughi University of Medical Sciences, Yazd, Iran; ${ }^{2}$ Reproductive and Genetic Unit, Recurrent Abortion Research Center, Yazd Reproductive Science Institute, Yazd University of Medical Sciences, Yazd, Iran; ${ }^{3}$ Clinical Research Development Center (CRDU), Qom University of Medical Sciences, Qom, Iran; ${ }^{4}$ Department of Medical Genetics, School of Medicine, Shahid Beheshti University of Medical Sciences, Tehran, Iran; ${ }^{5}$ Urogenital Stem Cell Research Center, Shahid Beheshti University of Medical Sciences, Tehran, Iran
Correspondence: Arezou Sayad; Mohammad Taheri

Department of Medical Genetics, School of Medicine, Shahid Beheshti University of Medical Sciences, PO Box 19857 17443,

Tehran, Iran

Tel/fax +98 2I 23872572

Email ar.sayad@yahoo.com; mohammad_823@yahoo.com
Introduction: Low level of vitamin D is a potential risk factor for developing schizophrenia. Through interaction with its receptor (VDR) and the related enzymes (CYP27B1, CYP24A1), vitamin $\mathrm{D}$ modulates neurodevelopment, neuroprotection, and immunomodulation. Its deficiency leads to aberrant neurodevelopment in schizophrenic patients.

Methods: In this case-control study, relative expression of VDR, CYP27B1, and CYP24A1 in schizophrenic patients was compared with healthy individuals. Total RNA was extracted from whole blood of 50 patients with schizophrenia and 50 healthy controls. Real-time PCR was used to determine relative gene expression levels of VDR, CYP27B1, and CYP24A1.

Results: Significant upregulations were observed in $V D R(P=0.004,95 \% \mathrm{CI}=0.77,0.86), C Y P 27 B 1$ $(P=0.002,95 \% \mathrm{CI}=1.22,4.98)$, and $C Y P 24 A 1(P \leq 0.0001,95 \% \mathrm{CI}=-2.721,1.061)$ expressions in peripheral blood of schizophrenic patients compared with controls. Moreover, the gender-based analysis revealed upregulation of all genes in all the categories of male and female except for $V D R$ gene in male group $(P=0.234,95 \% \mathrm{CI}=-0.79,3.35)$ and $C Y P 27 B 1$ gene in the female group $(P=0.09$, $95 \% \mathrm{CI}=-0.21,6.55)$. The age-based analysis demonstrated overexpression of $V D R$ and $C Y P 27 B 1$ genes in all categories. Finally, there were significant correlations between expression levels of all genes $(P<0.0001)$, while no correlation was found between age and expression of genes.

Conclusion: We hypothesized that the observed upregulation of the mentioned genes in schizophrenia patients might be the result of a compensatory mechanism to protect the affected individuals against adverse consequences of this disorder. Such imbalance in vitamin D processing pathway might also be implicated in the pathogenesis of schizophrenia. However, future studies should be designed to confirm the results of the current study.

Keywords: $C Y P 27 B 1, C Y P 24 A 1, V D R$, schizophrenia

\section{Introduction}

Schizophrenia is a debilitating psychiatric disorder with various etiologies affecting nearly $1 \%$ of the global population. ${ }^{1}$ It consists of three major disabling categories: positive, negative, and cognitive symptoms. ${ }^{2}$ Both environmental and genetic factors are involved in the etiology of this disorder. ${ }^{2}$ Meanwhile, environmental factor might also provoke the disease onset in genetically predisposed persons. Among environmental factors, vitamin D is of particular interest. This vitamin plays a significant role in neurodevelopment as well as immunomodulation. ${ }^{3}$ In the central nervous system (CNS), vitamin D contributes to both the synthesis of neurotransmitters and neuroprotection against damages and inflammation. ${ }^{3}$ It is also capable of protecting the brain against reactive oxygen species (ROS), through upregulation of some known antioxidant molecules such as glutathione (GSH). ${ }^{4}$ It has long been considered that prenatal exposure to low vitamin D may increase susceptibility to psychiatric disorders later in life. This finding is postulated by several epidemiological studies. ${ }^{5}$ The active 
form of vitamin D is produced through two hydroxylation steps in liver and kidney. In the kidney, CYP27B1, which is a member of CYP/CYP450 superfamily is responsible for $1 \alpha$ hydroxylation on 25 -hydroxy vitamin $\mathrm{D}_{3}\left(25 \mathrm{OHD}_{3}\right)$ to create the active form $1 \alpha 25$-dihydroxyvitaminD $\left(1 \alpha 25 \mathrm{OH}_{2} \mathrm{D}_{3}\right)$. It is worth mentioning that $1 \alpha 25 \mathrm{OH}_{2} \mathrm{D}_{3}$ can regulate its production via CYP24A1 (another member of CYP/CYP450 enzymes). The latter enzyme deactivates $1 \alpha 25 \mathrm{OH}_{2} \mathrm{D}_{3}$ through 24 oxidation pathway leading to the formation of calcitroic acid and lactone. ${ }^{6} 1 \alpha 25 \mathrm{OH}_{2} \mathrm{D}_{3}$ exerts its effects on different tissues by genomic and nongenomic pathways. Genomic pathway initiates when $1 \alpha 25 \mathrm{OH}_{2} \mathrm{D}_{3}$ binds to its receptor known as VDR. ${ }^{7}$ Thereby, it modulates the expression of several genes involved in diverse physiological functions such as calcium hemostasis, growth control, differentiation, cognition, and immune response. ${ }^{8}$ VDR is expressed in a wide variety of nerve cells and different regions of the nervous system. Moreover, hydroxylation process is not confined to kidney and liver, but it has been discovered in numerous other tissues. ${ }^{8,9}$ Overall, the importance of vitamin D and its metabolizing enzymes in the development of the nervous system are undeniable. Considering these and lack of studies related to the expression of genes involved in metabolism of vitamin D in schizophrenia, in this case-control study, we aimed to investigate the relative expression of $V D R$, $C Y P 24 A 1$, and CYP27B1 in peripheral blood of schizophrenic patients and healthy controls.

\section{Materials and methods Study design}

Clinical confirmation of schizophrenia was done according to the Diagnostic and Statistical Manual of Mental Disorders, Fifth Edition (DSM-V) by a certified psychiatrist. Controls were closely matched for age and gender with cases. The inclusion criteria for patients were defined as follows: definite diagnosis of the disorder based on DSM-V. ${ }^{10,11}$ Individuals recruited in control group were assessed through a structured psychiatric interview for ruling out the presence of any psychiatric disorder. Individuals who had the drug abuse, traumatic events, and severe medical conditions such as epilepsy and history of related disorders in first-degree relatives were excluded from the study. All the patients received Clozapine $^{\mathrm{TM}}$ as treatment. ${ }^{12}$

\section{Blood sampling}

In this study, $5 \mathrm{~mL}$ of peripheral blood was obtained from both groups. Written informed consent was obtained from all study participants. All procedures were performed with verification from the Ethics Committee of Shahid Beheshti University of Medical Sciences.

\section{Laboratory analysis}

Total RNA was isolated by Hybrid-RTM blood RNA extraction kit (cat no 305-101, GeneAll, Biotechnology Co. Ltd, Seoul, Korea). RNA integrity and purity were assessed by NanoDrop equipment. Low-quality RNA samples were excluded from the study. Purified RNA was stored at $-80^{\circ} \mathrm{C}$. In the succeeding step, the High-Capacity cDNA Reverse Transcription Kit (PN: 4,375,575, Applied Biosystems, Thermo Fisher Scientific, Waltham, MA, USA) was used for the synthesis of cDNA. All the procedures were performed according to the manufacturer's protocol. In order to perform TaqMan quantitative real-time PCR, the specific primers and probes were designed using Allele ID7 for x64 windows software (Premier Biosoft, Palo Alto, CA, USA). The specificity and validity of primers were determined by NCBI primer blast. The sequence of primers and probes are shown in Table S1. Finally, real-time PCR was carried out on a Corbett Rotor Gene 6000 system (Corbett Life Science, Sydney, Australia) with the TaqMan ${ }^{\circledR}$, Universal PCR Master Mix (PN: 4,304,449, Applied Biosystems). In order to control the quality of method, we included negative control. Moreover, HPRT1 was used as a reference gene in order to normalize relative expression of target genes. Each sample was analyzed in triplicate. Relative expression of genes in patients compared with healthy subjects was estimated based on calculation of $\mathrm{Ln}\left[\right.$ Efficiency ${ }^{\wedge}(\mathrm{CT}$ reference gene-CT target gene $\left.)\right]$ values.

\section{Statistical analysis}

The independent sample $t$-test was applied to examine the differences between two groups. The one-way ANOVA test was used to compare means between control and patient groups. Pearson's correlation coefficient was calculated to assess correlations between variables. The KolmogorovSmirnov test was used to investigate the normality. The level of significant $P$-value was set at $\leq 0.05$. Data analysis was performed using SPSS version 18 (Chicago, IL, USA).

\section{Result}

\section{Clinical and demographic information}

The demographic features of the study groups are summarized in Table 1. No significant difference was found in age of cases and controls $(P=0.0581)$ or in sex ratio $(P=0.18)$. All the individuals were compared in three ways: A) total participants together (regardless of age and sex), B) sexbased analysis (male or female), and C) age-based analysis 
Table I Demographic features of schizophrenia patients and healthy controls

\begin{tabular}{l|l|l}
\hline Variables & $\begin{array}{l}\text { Schizophrenia } \\
\text { patients }\end{array}$ & Control \\
\hline Female/male, $n(\%)$ & $15(30) / 35(70)$ & $18(36) / 32(64)$ \\
Age (years), mean \pm SD & $50.7 \pm 4.2$ & $49.2 \pm 3.6$ \\
Age range (years) & $30-69$ & $29-63$ \\
Male $<30$ & 7 & 8 \\
Female $<30$ & 0 & 4 \\
$30<$ male $<40$ & 13 & 8 \\
$30<$ female $<40$ & 4 & 7 \\
Male $>40$ & 15 & 16 \\
Female $>40$ & 11 & 7 \\
Age at onset (years), mean \pm SD & $35 \pm 1.2$ & - \\
Years of illness, mean \pm SD & $8 \pm 0.04$ & - \\
Education & & 12 \\
Preschool (\%) & 30 & 28 \\
School (\%) & 48 & 60 \\
University (\%) & 22 & \\
\hline
\end{tabular}

$(<30,30-40$, and $>40$ years $)$. Therefore, the group of schizophrenia patients was compared with controls, as well as each of the sex and age subgroup.

\section{Relative expression level of the VDR}

We observed upregulation of $V D R$ expression in the peripheral blood of schizophrenic patients compared with healthy controls $(P=0.004,95 \% \mathrm{CI}=0.77,0.86)$. In the gender-based analysis of $V D R$ expression, we detected a statistically significant change in expression levels of VDR in female affected by schizophrenia $(P<0.0001,95 \% \mathrm{CI}=3.17,6.15)$. Furthermore, schizophrenic females aged between 30 and 40 years and both male and female patients over the age of 40 years had a significant increase in expression of $V D R$ $(P=0.001,95 \% \mathrm{CI}=4.25,7.09 ; P=0.047,95 \% \mathrm{CI}=0.03,6.36$; and $P=0.03,95 \% \mathrm{CI}=1.15,6.83$, respectively) (Table 2).

\section{Relative expression level of the CYP27BI}

CYP27B1 total expression level was significantly higher in the peripheral blood of schizophrenic patients compared with controls $(P=0.002,95 \% \mathrm{CI}=1.22,4.98)$. Gender-based assessments revealed upregulation in both male and female groups. However, this increase was merely significant in the male group $(P=0.012,95 \% \mathrm{CI}=0.7,5.41)$. In addition, we observed $C Y P 27 B 1$ upregulation in schizophrenic male patients aged over 40 years compared with the corresponding control group $(P=0.01,95 \% \mathrm{CI}=1.32,8.7)$ (Table 2$)$.

\section{Relative expression level of the CYP24A I} CYP24A1 expression level was increased in the peripheral blood of schizophrenic patients compared with controls

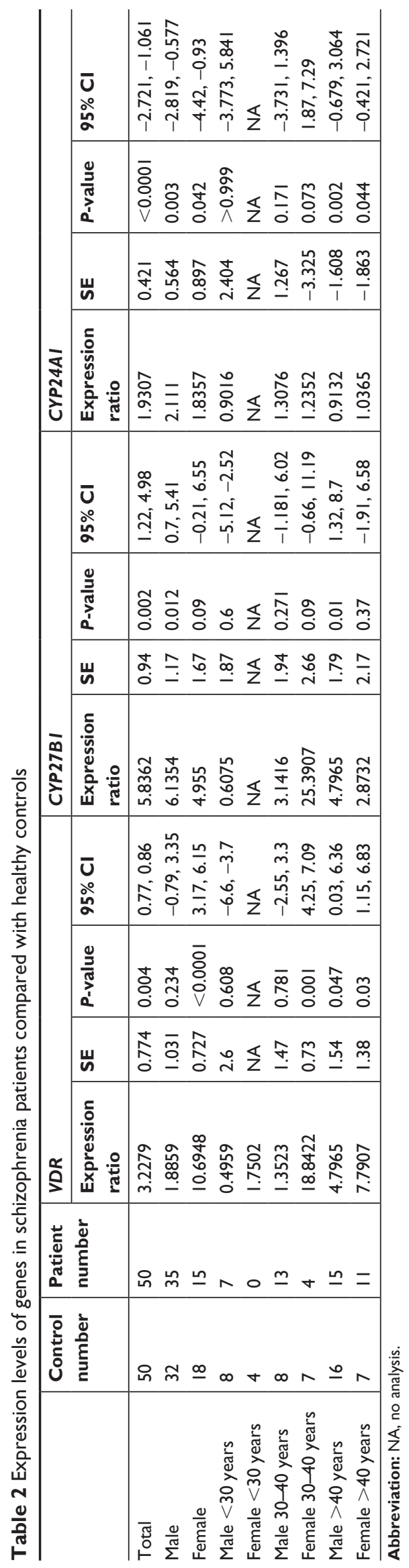


$(P<0.001,95 \% \mathrm{CI}=-2.721,-1.061)$. Such upregulation was also significant in male and female subgroups $(P=0.003$, $95 \% \mathrm{CI}=-2.819,-0.577$ and $P=0.042,95 \% \mathrm{CI}=-4.42,-0.93$, respectively). However, in the subgroup of patients aged over 40 years, both male and female patients demonstrated downregulation of $C Y P 24 A 1$ expression $(P=0.002,95 \%$ $\mathrm{CI}=-0.679,-3.064$ and $P=0.044,95 \% \mathrm{CI}=-0.421,-2.721$, respectively) (Table 2 ).

Correlation between the expression levels of VDR/ CYP27BI, VDR/CYP24AI, and CYP27BI/CYP24AI in schizophrenic patients and healthy controls

Correlations between expression levels of $V D R / C Y P 27 B 1$, $V D R / C Y P 24 A 1$, and $C Y P 27 B 1 / C Y P 24 A 1$ are demonstrated in Figure $1 \mathrm{~A}-\mathrm{C}$. All correlations were statistically significant $(P<0.0001)$.

\section{Correlation between expressions of vitamin}

\section{D-related genes and age of study participants}

Increase in the age of schizophrenia patients did not affect the expression level of the vitamin D-related genes. As in Figure $2 \mathrm{~A}-\mathrm{C}$, there were not any statistically significant correlation between expression of genes and age either in patients or healthy subjects $(P>0.05)$.
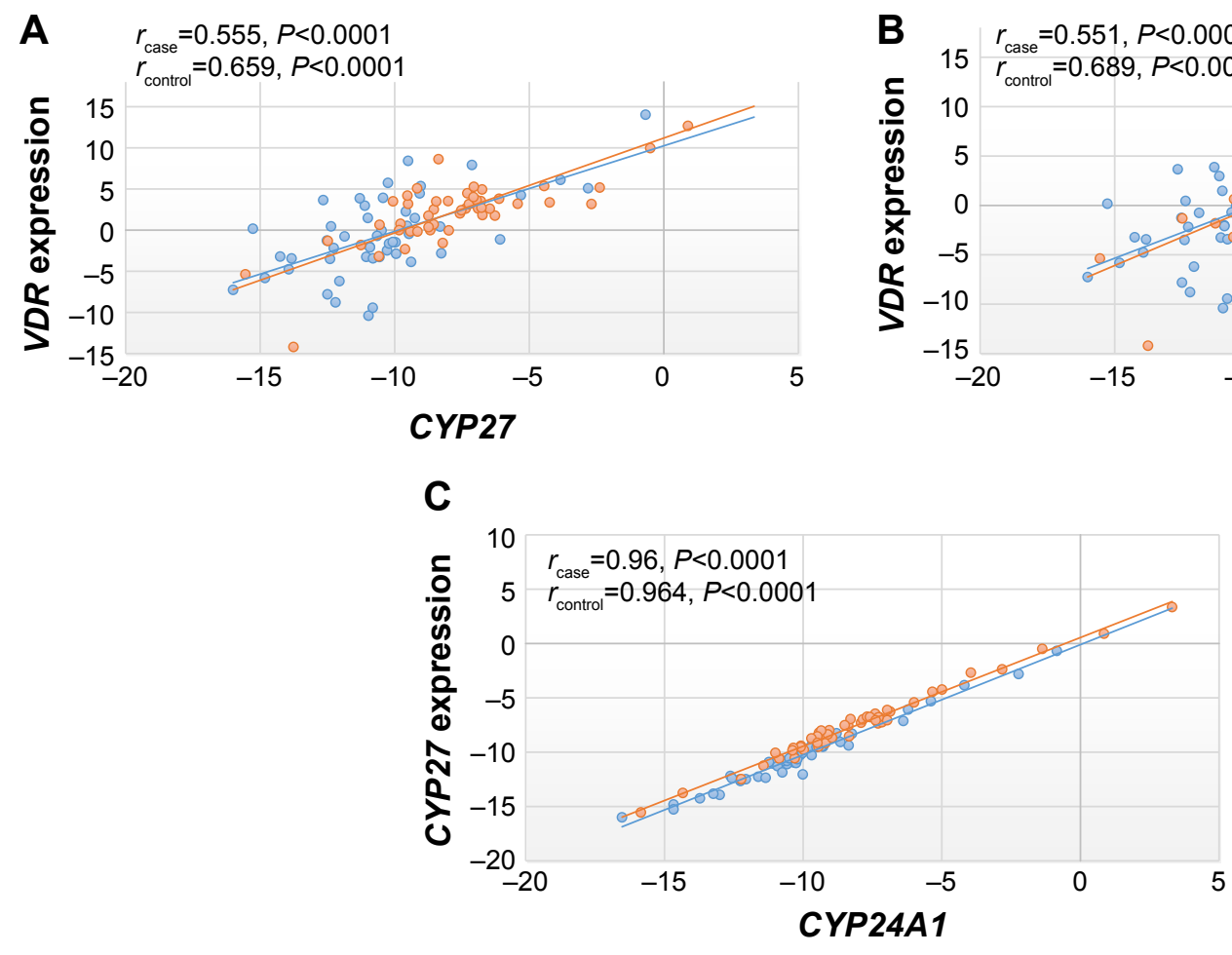

- Patient - Control — Linear (patient) — Linear (control)

Figure I (A) Correlation between VDR and CYP27 expressions. (B) Correlation between VDR and CYP24AI expressions. (C) Correlation between CYP27 and CYP24AI expressions.

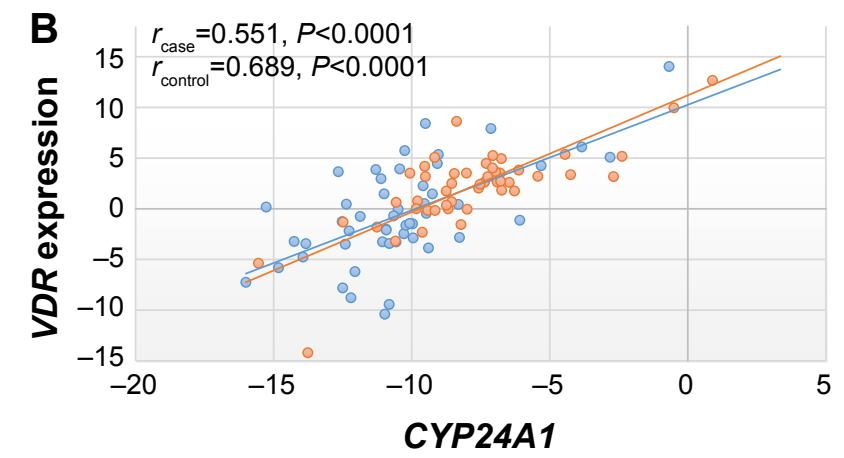

In this study, we compared the expression of vitamin Drelated genes in schizophrenic patients and healthy controls using quantitative real-time PCR. Our data revealed significant upregulation of $C Y P 27 B 1, V D R$, and $C Y P 24 A 1$ in schizophrenia patients compared with controls $(P=0.002$, $P=0.004$, and $P<0.0001$, respectively). In order to examine the clinical relevance of our results, we also assessed the expression of these genes in brain samples using the public available data of the SZDB database (http://www.szdb.org/). The dataset GSE12649 showed upregulation of VDR (fold change $=1.54, P=0.047$ with $204255 \_$s_at probe). However, expressions of the other genes were not different between cases and controls using the same dataset.

In schizophrenia, males are more affected than females. Several lines of evidence suggest that estrogen in female can have a protective role against neuropsychiatric diseases. Both estrogen and vitamin $\mathrm{D}$ activate $\mathrm{TPH}_{2}$ gene responsible for serotonin production. ${ }^{13-15}$ Therefore, we hypothesized that the change in expression ratio of VDR and CYP27B1 is more probable in male than female or it is plausible that male display alteration in vitamin $\mathrm{D}$ gene expression with more intensity. As a result, we carried out gender-based analysis. However, in contrast to our speculation, female 

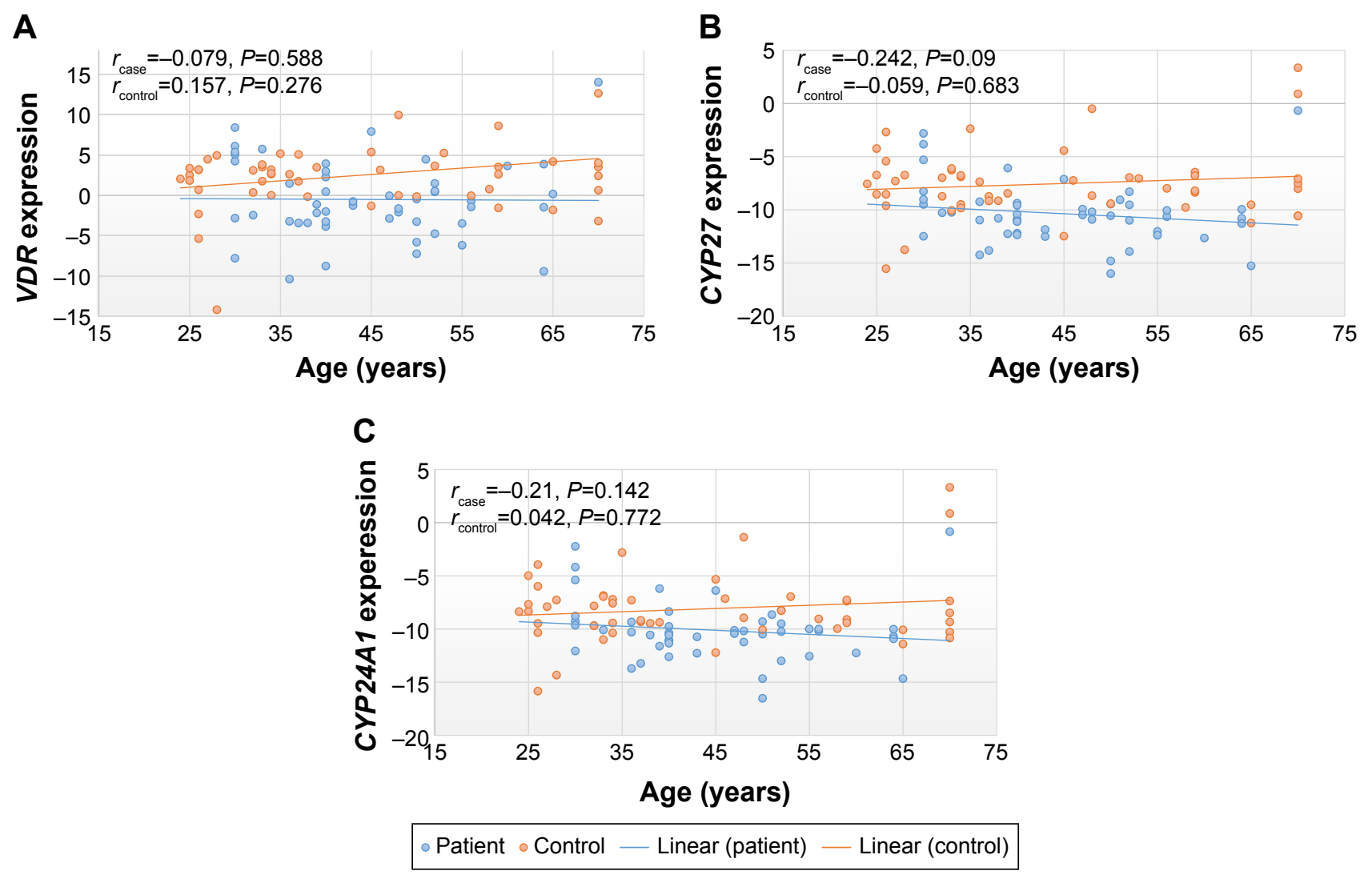

Figure 2 (A) Correlation between VDR expression and age. (B) Correlation between CYP27 expression and age. (C) Correlation between CYP24AI expression and age.

patients revealed a significant increase in $V D R$ expression $(P<0.0001)$. Furthermore, in $C Y P 27 B 1$ expression analysis, both male and female patients had considerable upregulation compared with healthy controls. However, only CYP27B1 upregulation in male category was statistically significant ( $P=0.012)$. In the age-based analysis, based on estrogen hypothesis that was mentioned earlier, we expected upregulation in VDR and CYP27B1 gene expression in affected female over 40 years old because these individuals have lower amounts of estrogen. For $V D R$ gene, these results were not compatible with the suggested hypothesis. For instance, both female and male over 40 years old showed increase in the $V D R$ expression ( $P=0.03$ and $P=0.04$, respectively), while female aged between 30 and 40 years also represented this increase $(P=0.001)$. $C Y P 27 B 1$ overexpression was only significant for schizophrenic male patients aged over 40 years $(P=0.01)$. CYP24A1 gene revealed downregulation in both male and female patients aged over 40 years $(P=0.002$ and $P=0.004$, respectively). To our knowledge, this was the first study evaluating the expression levels of VDR and related enzymes in schizophrenia. So far, most of the studies that have assessed vitamin D-related gene in schizophrenia have mainly focused on polymorphisms of these genes ${ }^{16,17}$ or assessed serum level of vitamin D in schizophrenic patients and reported lower serum level of vitamin D in these patients compared with controls. ${ }^{18}$ In this study, we also assessed the correlation between the expression of vitamin D-related genes and the age of study participants. Expressions of the assessed genes were not correlated with age either in schizophrenia patients or in controls, which is in line with the findings reported in multiple sclerosis patients..$^{19}$ On the contrary, all pairwise correlations between expression levels of genes were significant. In our study, schizophrenia patients had been treated with an equal dose of clozapine. Clozapine is an atypical drug, and its mechanism of action is not comprehensively known. It acts on histaminergic, muscarinic, adrenergic, and serotonin receptors. Gene expression studies using microarray in both rat and human postmortem brain showed that clozapine regulates several genes acting in various biological pathways. Genes that are modulated by this drug are suggested as candidate genes in schizophrenia. However, vitamin D signaling pathway was not among those possibly regulated pathways by clozapine. ${ }^{20,21}$ This may indicate that changes in vitamin $\mathrm{D}$ in schizophrenia can be the consequence of this disorder not the cause of it. Moreover, clozapine does not obviate the action of DHCR7 enzyme and does not alter the level of vitamin D precursor (7DHC) either in rat or in human. ${ }^{22}$ Overall, it is unlikely that change 
in VDR, CYP24A1, and CYP27B1 expression observed in schizophrenia patients in this study is the result of clozapine action on gene regulation. Our findings suggest that increased expression levels of $V D R$ and $C Y P 27 B 1$ may be involved in the pathophysiology of schizophrenia through various pathways. There are growing evidences linking vitamin D to neuroplasticity, neuroprotection, and neurotransmission. Low level of this vitamin is considered as a potential risk factor for developing psychiatric and neurological disorders. ${ }^{9}$ Vitamin D deficiency can cause long-term dysregulation in genes that are involved in mitochondrial function, cytoskeletal maintenance, and neurotransmission. ${ }^{5}$ Previous reports indicate the presence of vitamin $\mathrm{D}$ metabolite $25(\mathrm{OH}) \mathrm{D}$ in the cerebrospinal fluid (CSF) and the correlation between CSF and serum 25(OH)D levels. ${ }^{23}$ Besides, vitamin D and its carrier protein infiltrate to the $\mathrm{CNS} .{ }^{24}$ So, we suggest that the expression of metabolizing enzymes in the blood might affect vitamin D levels in the brain. However, future studies are needed to verify this hypothesis.

As mentioned earlier, schizophrenia is a disorder of heterogeneous nature. Several studies have concentrated on the dopaminergic hypothesis of schizophrenia. Nevertheless, in recent years, growing number of studies have focused on other aspects of this disorder such as neuroinflammation and redox dysregulation along with NMDA receptor (NMDAR) hypofunction. Redox dysregulation is observed in schizophrenia patients. ${ }^{25}$ The imbalance in the production of ROS and reactive nitrogen species along with deficiency in enzymatic and nonenzymatic antioxidants can lead to oxidative and nitrosative stress. ${ }^{25} \mathrm{GSH}$ is a cellular antioxidant. Its synthesis and metabolism are controlled by gamma-glutamyl transpeptidase (GGT). It is now postulated that the active form of vitamin D $(1,25 \mathrm{OH} 2 \mathrm{D} 3)$ upregulates activation and gene expression of GGT. Furthermore, 1,25OH2D3 increases activation of GSH reductase, GSH peroxidase, and glutamyl cysteine ligase catalytic subunit. ${ }^{26-29}$ Vitamin D serum level can elevate GSH expression in astrocytes. Additionally, diminished levels of GSH were observed in prefrontal cortex and CSF of schizophrenia patients and its lower levels associated with more severe negative symptoms..$^{2,26-28,30}$ Interestingly, this antioxidant agent has the capability to regulate different neurotransmitter systems such as NMDARs and GABA-A receptor. GSH deficiency as a result of redox dysregulation can lead to NMDAR hypofunction and decreased myelination

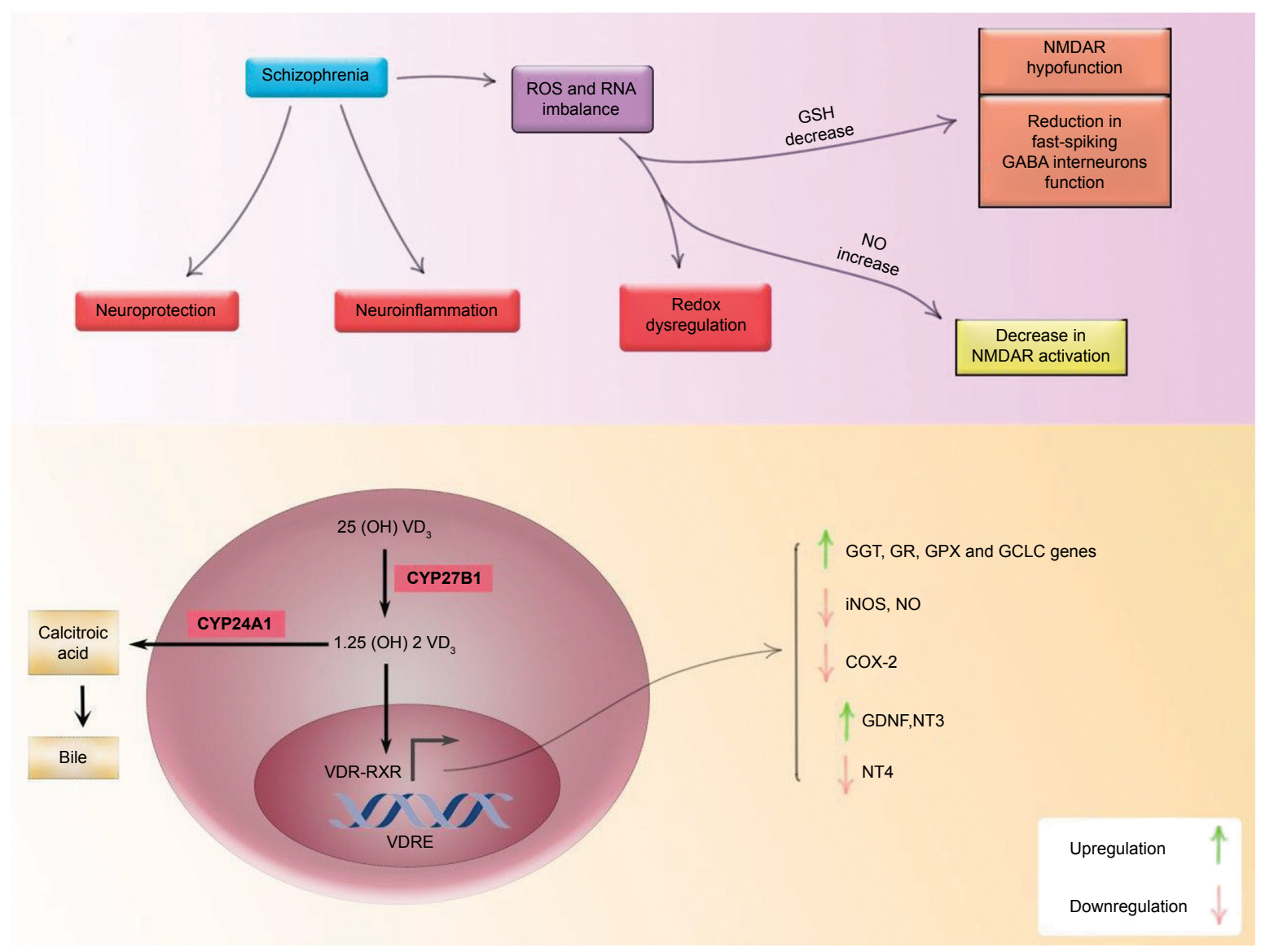

Figure 3 The role of vitamin D in four pathways involved in the etiology of schizophrenia. 
in schizophrenia patients, two conditions that can reduce fast-spiking GABA interneurons function causing negative, positive, and cognitive symptoms of schizophrenia. ${ }^{25,31}$ Based on the available evidence, low level of GSH can be a potential factor for NMDAR hypofunction, which is reported in schizophrenia. 1,25OH2D3 also acts as a neuroprotective agent through downregulation of inducible nitric oxide synthase (iNOS). ${ }^{2,30}$ This enzyme releases nitric oxide (NO) during the reaction. NO metabolites damage neurons as well as oligodendrocytes when they are produced in large amount. In postmortem studies, elevated levels of $\mathrm{NO}$ and NOS was identified in schizophrenic patients. ${ }^{26,32}$ There is also evidence indicating immune activation in schizophrenia. Inflammatory symptoms have been observed in postmortem studies in schizophrenia patients. As a result, inflammation might be a possible pathogenic mechanism in this disorder. COX2 (cyclooxygenase 2 is a rate-limiting enzyme in prostaglandin biosynthesis) mediates inflammatory response, and it is induced by cytokines such as IL2, IL6, and IL10. By recruiting genomic pathway, 1,25OH2D3 suppresses transcription of COX2 gene, which can lead to the succeeding inhibition of proinflammatory cytokines secretion. ${ }^{33,34} \mathrm{On}$ the contrary, 1,25OH2D3 works as a neurotrophic support through upregulation of GDNF (glial cell line-derived neurotrophic factor), NT3 (neurotrophin 3), and downregulation of NT4 (neurotrophin 4). ${ }^{35}$ Figure 3 has summarized the role of vitamin D in four pathways involved in the etiology of schizophrenia. CYP27B1 converts vitamin D to its active form $1,25 \mathrm{OH} 2 \mathrm{D} 3$, which is then bound to its receptor VDR. VDR heterodimerized with retinoid $\times$ receptor and modulates the expression of target genes. As mentioned above, it elevates GSH levels and upregulates mRNA expression of NGF, NT3, GDNF, and GGT, while it downregulates inflammation and mRNA expression of iNOS, COX2, and NT4 in astrocytes and microglia. CYP24A1 degrades this active form and terminates its effects. ${ }^{30}$ In this study, we observed approximately two-fold increase in CYP24A1 expression level, which can be justified by positive feedback through VDR as a result of increase in CYP27B1. Moreover, we found that patients with schizophrenia had significant upregulation in $V D R$ and $C Y P 27 B 1$ gene expression. Therefore, we hypothesized that these findings might be a consequence of schizophrenic disorder in order to apply the compensatory mechanism to promote antioxidant protection against oxidative and nitrosative stress as well as alleviating neuroinflammation and boosting neurotrophic support in patients affected by schizophrenia. Finally, the main limitation of this study is the lack of first-episode schizophrenia patients in order to evaluate the gene expression free of possible confounding effects of medication. Moreover, we did not have information about vitamin D status of study participants, and we did not have access to postmortem brain tissues to evaluate expression of genes in them.

\section{Conclusion}

Schizophrenia is a severe mental condition with a diversified set of pathologies such as perturbed dopamine and glutamate pathways, aberrant redox regulation, and neuroinflammation, all of which create a common central pathway. ${ }^{29}$ The active form of vitamin $\mathrm{D}$ upregulates or downregulates numerous factors acting in these pathways. Both VDR coding mRNA and its protein are present in the CNS. The mRNA expression of VDR gene has been identified both in neuronal and glial cells. Our findings suggest that increased mRNA expression of $V D R$ and $C Y P 27 B 1$ may be involved in the modulation of mentioned pathways through the genomic mechanism in order to ameliorate symptoms of the disease. Further investigations are needed to support this claim and determine the exact regulatory mechanism of vitamin $\mathrm{D}$ in each pathway. It is also valuable to measure the expression levels of VDR and related enzymes simultaneously with that of other molecular agents involved in oxidative, nitrosative stress, immune activation, and neurotrophic support in schizophrenia patients.

\section{Acknowledgments}

The authors would like to thank the schizophrenic patients and the Schizophrenia Society of Iran for their kind contribution in conducting this study. This study was supported technically and financially by Shahid Beheshti University of Medical Sciences.

\section{Disclosure}

The authors report no conflicts of interest in this work.

\section{References}

1. Escudero I, Johnstone M. Genetics of schizophrenia. Curr Psychiatry Rep. 2014;16(11):502.

2. Amaral AD, Calhau C, Coelho R. Schizophrenia: implications of vitamin D deficit on brain development. IJCNMH. 2014;1(16):1-14.

3. Cieslak K, Feingold J, Antonius D, et al. Low vitamin D levels predict clinical features of schizophrenia. Schizophr Res. 2014;159(2-3): 543-545.

4. Yüksel RN, Altunsoy N, Tikir B, et al. Correlation between total vitamin D levels and psychotic psychopathology in patients with schizophrenia: therapeutic implications for add-on vitamin D augmentation. Ther Adv Psychopharmacol. 2014;4(6):268-275.

5. Eyles D, Almeras L, Benech P, et al. Developmental vitamin D deficiency alters the expression of genes encoding mitochondrial, cytoskeletal and synaptic proteins in the adult rat brain. J Steroid Biochem Mol Biol. 2007;103(3-5):538-545.

6. Prosser DE, Jones G. Enzymes involved in the activation and inactivation of vitamin D. Trends Biochem Sci. 2004;29(12):664-673. 
7. Groves NJ, McGrath JJ, Burne TH. Vitamin D as a neurosteroid affecting the developing and adult brain. Annu Rev Nutr. 2014;34: 117-141.

8. Schuster I. Cytochromes P450 are essential players in the vitamin D signaling system. Biochim Biophys Acta. 2011;1814(1):186-199.

9. Deluca GC, Kimball SM, Kolasinski J, Ramagopalan SV, Ebers GC. Review: the role of vitamin D in nervous system health and disease. Neuropathol Appl Neurobiol. 2013;39(5):458-484.

10. Rahimi S, Sayad A, Moslemi E, Ghafouri-Fard S, Taheri M. Blood assessment of the expression levels of matrix metalloproteinase 9 (MMP9) and its natural inhibitor, TIMP1 genes in Iranian schizophrenic patients. Metab Brain Dis. 2017;32(5):1537-1542.

11. Azimi T, Ghafouri-Fard S, Davood Omrani M, et al. Vaccinia related kinase 2 (VRK2) expression in neurological disorders: schizophrenia, epilepsy and multiple sclerosis. Mult Scler Relat Disord. 2018; 19:15-19.

12. Nafisi-Far N, Ghafouri-Fard S, Panah AST, Sayad A, Taheri M. A gender dimorphism in up-regulation of BACE1 gene expression in schizophrenia. Metab Brain Dis. 2018;33(3):933-937.

13. Kulkarni J, Riedel A, de Castella AR, et al. Estrogen - a potential treatment for schizophrenia. Schizophr Res. 2001;48(1):137-144.

14. Patrick RP, Ames BN. Vitamin D hormone regulates serotonin synthesis. Part 1: relevance for autism. FASEB J. 2014;28(6):2398-2413.

15. Patrick RP, Ames BN. Vitamin D and the omega-3 fatty acids control serotonin synthesis and action, part 2: relevance for ADHD, bipolar disorder, schizophrenia, and impulsive behavior. FASEB J. 2015;29(6):2207-2222.

16. Kuningas M, Mooijaart SP, Jolles J, Slagboom PE, Westendorp RG, van Heemst D. VDR gene variants associate with cognitive function and depressive symptoms in old age. Neurobiol Aging. 2009;30(3): 466-473.

17. Ahmadi S, Mirzaei K, Hossein-Nezhad A, Shariati G. Vitamin D receptor FokI genotype may modify the susceptibility to schizophrenia and bipolar mood disorder by regulation of dopamine D1 receptor gene expression. Minerva Med. 2012;103(5):383-391.

18. Nerhus M, Berg AO, Kvitland LR, et al. Low vitamin D is associated with negative and depressive symptoms in psychotic disorders. Schizophr Res. 2016;178(1-3):44-49.

19. Smolders J, Thewissen M, Theunissen R, et al. Vitamin D-related gene expression profiles in immune cells of patients with relapsing remitting multiple sclerosis. J Neuroimmunol. 2011;235(1-2):91-97.

20. Fatemi SH, Folsom TD, Reutiman TJ, Novak J, Engel RH. Comparative gene expression study of the chronic exposure to clozapine and haloperidol in rat frontal cortex. Schizophr Res. 2012;134(2-3): 211-218.
21. Lee BJ, Marchionni L, Andrews CE, et al. Analysis of differential gene expression mediated by clozapine in human postmortem brains. Schizophr Res. 2017;185:58-66.

22. Korade Ž, Liu W, Warren EB, Armstrong K, Porter NA, Konradi C. Effect of psychotropic drug treatment on sterol metabolism. Schizophr Res. 2017;187:74-81.

23. Holmøy T, Moen SM, Gundersen TA, et al. 25-hydroxyvitamin D in cerebrospinal fluid during relapse and remission of multiple sclerosis. Mult Scler. 2009;15(11):1280-1285.

24. Smolders J, Schuurman KG, van Strien ME, et al. Expression of vitamin D receptor and metabolizing enzymes in multiple sclerosisaffected brain tissue. J Neuropathol Exp Neurol. 2013;72(2):91-105.

25. Do KQ, Cabungcal JH, Frank A, Steullet P, Cuenod M. Redox dysregulation, neurodevelopment, and schizophrenia. Curr Opin Neurobiol. 2009;19(2):220-230.

26. Berridge MJ. Vitamin D cell signalling in health and disease. Biochem Biophys Res Commun. 2015;460(1):53-71.

27. Garcion E, Sindji L, Leblondel G, Brachet P, Darcy F. 1,25-dihydroxyvitamin D3 regulates the synthesis of gamma-glutamyl transpeptidase and glutathione levels in rat primary astrocytes. $J$ Neurochem. 1999;73(2):859-866.

28. Koga M, Serritella AV, Sawa A, Sedlak TW. Implications for reactive oxygen species in schizophrenia pathogenesis. Schizophr Res. 2016;176(1): 52-71.

29. Steullet P, Cabungcal J, Monin A, et al. Redox dysregulation, neuroinflammation, and NMDA receptor hypofunction: a "central hub" in schizophrenia pathophysiology? Schizophr Res. 2016;176(1):41-51.

30. Garcion E, Wion-Barbot N, Montero-Menei CN, Berger F, Wion D. New clues about vitamin D functions in the nervous system. Trends Endocrinol Metab. 2002;13(3):100-105.

31. Snyder MA, Gao WJ. NMDA hypofunction as a convergence point for progression and symptoms of schizophrenia. Front Cell Neurosci. 2013;7:31.

32. Bitanihirwe BK, Woo TU. Oxidative stress in schizophrenia: an integrated approach. Neurosci Biobehav Rev. 2011;35(3):878-893.

33. Müller N, Ulmschneider M, Scheppach C, et al. COX-2 inhibition as a treatment approach in schizophrenia: immunological considerations and clinical effects of celecoxib add-on therapy. Eur Arch Psychiatry Clin Neurosci. 2004;254(1):14-22.

34. Wang Q, He Y, Shen Y, et al. Vitamin D inhibits COX-2 expression and inflammatory response by targeting thioesterase superfamily member 4 . J Biol Chem. 2014;289(17):11681-11694.

35. Krivoy A, Hochman E, Sendt KV, et al. Association between serum levels of glutamate and neurotrophic factors and response to clozapine treatment. Schizophr Res. 2018;192:226-231. 


\section{Supplementary material}

Table SI The sequences of probes and primers

\begin{tabular}{lll}
\hline Property & VDR & HPRTI \\
\hline F-primer & TGGCTTTCACTTCAATGCTATGA & AGCCTAAGATGAGAGTTC \\
R-primer & CGTCGGTTGTCCTTGGTGAT & CACAGAACTAGAACATTGATA \\
Probe & FAM-ACTTCCGGCCTCCAGTTCGTATGGAC-TAMRA & FAM -CATCTGGAGTCCTATTGACATCGC- TAMRA \\
\hline Property & CYP27BI & CYP24AI \\
\hline F-primer & CCCAGATCCTAACACATTTTGAGG & TATCGCGACTACCGCAAAGA \\
R-primer & AAAGGGTGATGATGACAGTCTCTTTC & CGGCCAAGACCTCATTGATT \\
Probe & FAM-ACCCAAGACCCGGACTGTCCTGGT -TAMRA & FAM-TCCGGACCCGCTGCCAGTCTT -TAMRA \\
\hline
\end{tabular}

Abbreviations: F, forward; R, reverse.

\section{Publish your work in this journal}

Neuropsychiatric Disease and Treatment is an international, peerreviewed journal of clinical therapeutics and pharmacology focusing on concise rapid reporting of clinical or pre-clinical studies on a range of neuropsychiatric and neurological disorders. This journal is indexed on PubMed Central, the 'PsycINFO' database and CAS, and is the official journal of The International Neuropsychiatric Association (INA). The manuscript management system is completely online and includes a very quick and fair peer-review system, which is all easy to use. Visit http://www.dovepress.com/testimonials.php to read real quotes from published authors.

\footnotetext{
Submit your manuscript here: http://www.dovepress.com/neuropsychiatric-disease-and-treatment-journal
} 